\title{
Legitimate to extrapolate efficacy from one pain context to another
}

\author{
Is the third molar pain model different from other models and does it effect \\ analgesic response?
}

\begin{abstract}
Barden J, Edwards JE, McQuay HJ, Moore A. Pain and analgesic response after third molar extraction and other postsurgical pain. Pain 2004; 107:86-90

Data sources Sources of literature were Medline, Embase, the Cochrane Library, Biological Abstracts and the Oxford Pain Relief Database. Study selection Randomised, double-blind trials were chosen that compared aspirin, ibuprofen or paracetamol with placebo in adult patients who had initially moderate or severe postoperative pain, and which measured pain intensity and relief using standard measurement tools over 4-6h.
\end{abstract}

Data extraction and synthesis Criteria for inclusion were: full peer-reviewed journal publication; randomised controlled trials that included single-dose treatment groups of aspirin, ibuprofen or paracetamol and placebo; double-blind design; baseline pain of moderate to severe intensity; patients over 15 years of age; at least 10 patients per group; and the pain outcome measures of total pain relief (TOTPAR) or summed pain intensity difference (SPID) over 4-6h, or sufficient data to allow their calculation. Pain measures permitted for the calculation of TOTPAR or SPID were a standard five-point pain relief scale (none, slight, moderate, good, complete), a standard four-point pain intensity scale (none, mild, moderate, severe) or a standard visual analogue scale (VAS) for pain relief or pain intensity. Also accepted were the top two categories of a standard five-point global outcome scale (poor, fair, good, very good, excellent).

Results Results from 160 comparisons of an analgesic versus placebo were included. These comprised 68 trials of aspirin at doses of $600 /$ $650 \mathrm{mg} ; 49$ trials of ibuprofen at a dose of $400 \mathrm{mg} ; 19$ trials of paracetamol at $600 / 650 \mathrm{mg}$; and 24 trials of paracetamol at $1000 \mathrm{mg}$. Dental studies predominated, forming 102 of the 160 studies (64\%). There were no consistent differences between dental and postsurgical pain in single-dose trials of aspirin, either of two doses of paracetamol, or ibuprofen, where sufficiently large data sets exist to test the hypothesis. Only three out of 16 comparisons produced a statistically significant difference.

Conclusions A systematic difference in the estimate of analgesic efficacy between dental and postsurgical pain models remains unproven and, on balance, no major difference is likely.

\section{Commentary}

A major assumption in systematic reviews is that it is appropriate to pool results from similar studies in order to achieve higher levels of accuracy and precision in calculating the relative merit of therapeutic interventions. Yet how do we define 'similar'? For example, do the results from clinical trials of analgesics in patients with cancer pain offer value when considering treatment of pain in patients who have trigeminal neuralgia? One concept that has emerged from preclinical research is that pain conditions with similar biological mechanisms may exhibit similar pharmacology even though the measured outcome is different. For example, the relative potency of nonsteroidal anti-inflammatory drugs for reducing oedema in inflamed rat hindpaws is strikingly similar to their relative analgesic potency for treating patients with arthritis. ${ }^{1}$ The explanation for this observation, of course, is that the outcome measured in both biological models is due largely to the release of prostaglandins.

In this study, Barden and colleagues have analysed systematic reviews evaluating single-dose aspirin, ibuprofen and paracetamol in clinical models of acute pain resulting from the surgical extraction of third molars, episiotomy, and gynaecological, urological and other procedures. Because of the varied models, the study compared third molar extraction results with all other surgical procedures. This is perhaps one limitation from the present study since grouping disparate clinical models might increase variance and obscure potential differences. A subset analysis comparing third molar extraction with the largest available group of other surgical models might provide some information to deal with this concern. At least some of the data are suggestive of differences between third molar studies and the collection of 'surgery' studies since the placebo response was approximately half that observed in the former model compared with the latter models.

Overall, several conclusions can be reached from this analysis. First, post-surgical pain appears to be similar in terms of sensitivity to non-narcotic analgesics, without large differences observed across different body regions or after procedures varying in soft tissue/hard tissue disruption. This is important in being able to rely on summary tables giving relative benefits of non-narcotic analgesics across several models of acute surgical pain (for example, see www.jr2.ox.ac.uk/bandolier/booth/painpag/Acutrev/ Analgesics/Leagtab.html). Second, these similarities suggest that surgical pain models share common pain mechanisms that are modulated by non-narcotic analgesics. Thus, basic research into mechanisms of oral surgery pain may have strong implications across medicine and dentistry. Third, this type of study would be useful in evaluating other analgesics - for example, are opioids similar across clinical models? - or other models that appear to be based on more persistent forms of immune-derived inflammation, such as endodontic pain or rheumatoid arthritis.

\section{Practice point}

- Post-surgical pain in different areas of the body appears to be similar in terms of sensitivity to non-narcotic analgesics. Therefore, dentists can rely on summary tables produced using several models of acute surgical pain.

\section{Kenneth M Hargreaves}

Department of Endodontics, University of Texas Health Science

Center at San Antonio, San Antonio, Texas, USA

1. Otterness LG, Bliven ML. Laboratory models for testing non-steroidal antiinflammatory drugs. In Nonsteroidal Anti-inflammatory Drugs. Edited by Lombardino J. New York: Wiley; 1985;112-252. 